\title{
Startup Strategy Design and Safeguarding of Industrial Adiabatic Tubular Reactor Systems
}

\author{
J. W. Verwijs and H. van den Berg \\ Process Development \& Control Dept., Dow Benelux N.V., 4530 AA Terneuzen, The Netherlands \\ K. R. Westerterp \\ Chemical Reaction Engineering Laboratories, Dept. of Chemical Engineering, \\ Twente University, 7500 AE Enschede, The Netherlands
}

\begin{abstract}
The safeguarding methodology of chemical plants is usually based on controlling the instantaneous values of process state variables within a certain operating window, the process being brought to shutdown when operating constraints are exceeded. This method does not necessarily prevent chemical reactors suffering from a nunaway during dynamic operations because (a) excessive amounts of unreacted chemicals can still accumulate in the process, and (b) no means are provided to the operating personnel to identify hazardous process deviations. A model-based startup and safeguarding procedure is developed for an industrial adiabatic tubular reactor to improve process safety during startup. The trajectories of manipulated variables are calculated by minimizing the amount of one of the main reactants in the reactor effluent. It is concluded that proper control of the initial reactor temperature profile is critical for a safe startup while the impact of other manipulated variables is relatively smaller than that of the initial reactor temperature profile.
\end{abstract}

\section{Introduction}

Chemical reactors are more frequently involved in serious incidents than other types of process equipment (Garrison, 1988). The incident frequency in, for example, the United Kingdom only equals about two thermal runaway reactions per month for batch and semibatch reactor systems (Etchells, 1993) on a total inventory of about 2100 reactors (Marss et al., 1989). The severity of these incidents ranged from a controlled venting of the reaction mass to a violent loss of containment followed by an explosion of the released material (Barton and Nolan, 1984, 1989; Nolan and Barton, 1987). Hence, many studies have been carried out on reactive chemicals hazard assessment for batch and semibatch processes, resulting in guidelines for process design, control and operation (Barton and Rogers, 1993).

The research for the safe operation of continuously operated tubular reactor systems has almost been limited to thermal runaway prevention in (cooled) fixed-bed reactors. It resulted in design criteria to detect operating regions of para-

Correspondence concerning this article should be addressed to K. R. Westerterp. metrically sensitive behavior. In this domain the works of Hosten and Froment (1986), Morbidelli and Varma (1989), Bauman et al. (1990), and Raghaven (1992) can be mentioned without being exhaustive. In parametrically sensitive operating regions, relatively small changes in reactor inlet conditions or other physical parameters can lead to large excursions in the operating conditions ( = runaway behavior).

The direct implications of these parametric sensitivity criteria on the safe operation of industrial reactors are not obvious because the criteria are based on a parameter-sensitivity analysis of a (steady-state) reactor model and do not take the impact of the process dynamics and/or process abnormalities during the runaway into account (Verwijs et al., 1995b). Secondly, the research on runaway phenomena in (cooled) fixedbed tubular reactors is limited to model calculations only. A comparison of the theoretical results with experimental data from industrial reactors is not available, because too few industrial incidents have been thoroughly documented in the open literature for research purposes. Thirdly, the operation of continuously operated reactor systems is strongly interrelated with other process units. This tight coupling of process 
functions may make the entire plant more difficult to control, it complicates dynamic reactor operations like startup and shutdown (Verwijs et al., 1995a), and it has an impact on process safety. The latter aspect can be illustrated by the accident statistics of continuously operated chemical plants: the incident frequency, measured on a time basis, is much higher during startup and shutdown operations than at normal, steady-state operating conditions (Haastrup, 1983; Bhola, 1985; Garrison, 1988). Finally, the process safeguarding methodology currently used in the chemical industry is based on controlling the instantaneous values of the process state variables within a certain operating window, the process being brought to shutdown when the operating constraints are exceeded. Verwijs et al. (1995b) concluded from an analysis of runaways that occurred in industrial reactors that this safeguarding methodology does not necessarily prevent reactor systems from suffering a runaway because (a) excessive amounts of unreacted chemicals can still be present or accumulate in the process, and (b) no adequate means are provided to the operating personnel to identify hazardous process deviations during startup, production rate changes, or product grade changeovers.

In this article the startup behavior of an industrial adiabatic tubular reactor is studied in order to improve the process safeguarding methodology of the system. A dynamic model that describes the startup behavior of this industrial reactor has been reported by Verwijs et al. (1992). Some heuristic rules for the operation of an adiabatic tubular reactor during a plant startup were developed as well (Verwijs et al., 1995a), because the startup of a continuously operated reactor cannot be studied effectively without taking into account (qualitatively) the operational aspects of the entire plant section. In this study both the dynamic reactor model and the developed plant startup rules are used to design (quantitatively) an improved startup and safeguarding procedure for the reactor. The engineering principles used in this analysis are elementary for many continuously operated chemical plants. As a result, the proposed startup and safeguarding methodology can be extended to other cases relatively easily.

\section{Plant Reactor}

The plant reactor studied is shown in Figure 1, together with its upstream and downstream process sections. The process is shown in a simplified non-heat-integrated form in order to not reveal confidential information. In this reactor the products, denoted by $C$, are formed by an exothermal liquid phase reaction between the reactants $A$ and $B$ :

$$
A+B \rightarrow C \text {. }
$$

Reactant $A$ is fed from storage into a buffer tank, which also receives recycled material from a recovery unit. Reactant $A$ is fed from the buffer tank into the reactor together with reactant $B$, which is fed from storage. The adiabatic reactor system consists of a feed mixer, a preheater, and a series of seven vessels with baffles, of which only one vessel is shown in Figure 1 for the sake of simplicity; see Verwijs et al. (1992) for a detailed description of this reactor system. The per-

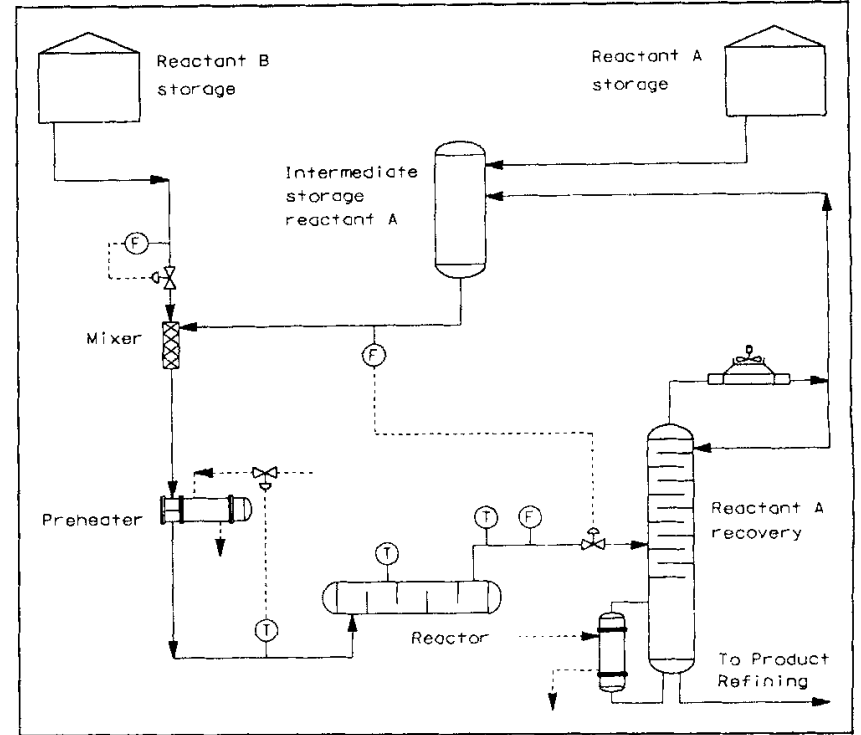

Figure 1. Process scheme.

formance of this reactor indicates that it can be characterized as a tubular reactor. Reactant $A$ is fed in excess, because reactant $B$ should be "totally" converted at the reactor exit to avoid the formation of reactive chemical mixtures elsewhere in the process. Note that total conversion of reactant $B$ in this context means that this component may appear only at very low concentration levels in the reactor effluent. The excess amount of reactant $A$ is recovered from the reactor effluent and recirculated. The entire reactant $A$ recovery section is represented by a single distillation column in Figure 1 . The crude product is discharged from the recovery unit into the product refining section.

The reactant $B$ flow rate is controlled by a valve in the $B$ feed line at a setpoint defined by a production capacity target. The reactant $A$ flow is controlled in ratio with the reactant $B$ flow by a valve in the reactor effluent line. The reactor effluent flow rate is recorded as well. The reactor inlet temperature is controlled at a setpoint that corresponds with the total feed rate into the reactor by a valve in the steam supply line to the preheater. The temperature along the reactor is recorded by thermoelements that are located at several positions between the reactor inlet $(z=0)$ and outlet $(z=1)$. The locations of the control valves, the flow devices $(F)$, and some thermoelements $(T)$ are indicated as well in Figure 1.

\section{Reactor Model}

Verwijs et al. (1992) presented a dynamic model of this industrial adiabatic tubular reactor in which the total reaction scheme had been simplified to one reaction, describing the consumption of reactant $B$. This reaction is assumed to be irreversible and of first order with an Arrhenius-type rate constant. The deviation of plug flow in the reactor was described by axial dispersion. All physical properties of the fluid were assumed to be constant over the entire reactor length. The energy take-up in the reactor vessel is included in the model, but the heat take-up in the insulation blanket and heat losses to the surroundings were neglected. The following dimensionless equations describe the reactant $B$ concentra- 
Table 1. Model Parameter Values

\begin{tabular}{cc}
\hline Model Parameter & Value \\
\hline$\gamma$ & 20.2 \\
$D a_{r}$ & 0.41 \\
$P e_{m r}$ & 100.0 \\
$P e_{h r}$ & 100.0 \\
$U^{*}$ & 160.0 \\
$\omega_{h}$ & 11.67 \\
$\Delta \theta_{a d r}$ & 0.34 \\
\hline
\end{tabular}

tion $\Gamma_{B}$, the reactor temperature $\theta$, and the reactor wall temperature $\theta_{w}$ as a function of the dimensionless time $\sigma$ and the axial reactor position $z$. All variables not defined in the text are defined in the Notation section. The values of the model parameters are listed in Table 1.

Mass Balance of Component B:

$$
\frac{\partial \Gamma_{B}}{\partial \sigma}=-\phi_{\nu} \frac{\partial \Gamma_{B}}{\partial z}+\frac{1}{P e_{m r}} \frac{\partial^{2} \Gamma_{B}}{\partial z^{2}}-D a_{r} \kappa \Gamma_{B} .
$$

\section{Energy Balance for the Fluid:}

$$
\begin{aligned}
\frac{\partial \theta}{\partial \sigma}=-\phi_{\nu} \frac{\partial \theta}{\partial z}+\frac{1}{P e_{h r}} \frac{\partial^{2} \theta}{\partial z^{2}}-D a_{r} U^{*}( & \left.\theta \theta_{w}\right) \\
& +\Delta \theta_{a d r} D a_{r} \kappa \Gamma_{B} .
\end{aligned}
$$

Energy Balance for the Reactor Vessel:

$$
\frac{\partial \theta_{w}}{\partial \sigma}=D a_{r} U^{*} \omega_{h}\left(\theta-\theta_{w}\right)
$$

These partial differential equations are subject to the following initial and boundary conditions:

Initial Conditions:

$$
\begin{array}{ll}
\sigma=0 ; & \Gamma_{B}(z, \sigma)=\Gamma_{B}(z, 0)=0 \\
\theta(z, \sigma) & =\theta(z, 0) \\
& \theta_{w}(z, \sigma)=\theta_{w}(z, 0)=\theta(z, 0) .
\end{array}
$$

\section{Boundary Conditions:}

$$
\begin{array}{ll}
\sigma \geq 0 ; \quad \text { and } \quad z=0 \quad \Gamma_{B}(0, \sigma)=\Gamma_{B 0}(\sigma)=\psi_{B} \\
& \theta(0, \sigma)=\theta_{0}(\sigma)=\nu_{\theta} \\
\sigma \geq 0 \text { and } \quad z=1 ; & \frac{\partial^{2} \Gamma_{B}}{\partial z^{2}}=\frac{\partial^{2} \theta}{\partial z^{2}}=0 .
\end{array}
$$

The boundary conditions applied at the reactor inlet and outlet are approximations of the continuity equations. These approximations are used to reduce computer time, because the impact of more sophisticated boundary conditions on the final results will be negligible since the plant reactor operates at relatively high Péclet numbers (Verwijs et al., 1992). Equations 1,2 , and 3 represent the system response as a function of the superficial fluid velocity $\phi_{\nu}$, the reactant $B$ inlet concentration $\psi_{B}$, and the reactor inlet temperature $\nu_{\theta}$, starting from the initial conditions at time $\sigma=0$ as a reference point. Hence, the startup behavior of the tubular reactor can be controlled by manipulating the following:

- The initial reactor temperature profile:

$$
\theta(z, \sigma)=\theta(z, 0)
$$

- The reactant $B$ inlet concentration $\psi_{B}$, which for this particular reactor system can be calculated from the reactant $A$ and $B$ flow rates $F_{A}$ and $F_{B}$ according to:

$$
\psi_{B}=\frac{4.0 \cdot F_{B}}{\left(2.62 \cdot F_{A}+F_{B}\right)}
$$

- The total flow rate $\phi_{v}$ through the system, which can be calculated from the reactant $A$ and $B$ flow rates according to:

$$
\phi_{v}=0.0112 \cdot F_{A}+0.0043 \cdot F_{B} .
$$

- The reactor inlet temperature $\nu_{\theta}$, which is equivalent to

$$
\nu_{\theta}=\theta(0, \sigma)
$$

The reactant flow rates $F_{A}$ and $F_{B}$ are expressed as a percentage of the particular flow device ranges to not reveal confidential information. The numbers shown in Eq. 8 and 9 are calculated accordingly.

\section{Reactor Startup Strategy}

Verwijs et al. (1995a) presented a sequence of intermediate operating states for the start-up of a continuously operated adiabatic tubular reactor by taking into account the operational aspects of an entire process section. Four consecutive steps are determined to drive the reactor system from shutdown to the normal production state:

- First, reactant $A$ should be recycled over the entire process section. This process recycle loop should include the reactor feed system, the reactor, and the recovery system.

- Second, the reactant $A$ recovery system should be driven to production state conditions, so the recovery system can treat the reactor effluent without significant process upsets after the start-up of the reactant $B$ feed into the reactor.

- Third, the reactor temperature should be driven to the required initial temperature $\theta(z, 0)$ at which reactant $B$ can be fed into the reactor safely (the so-called process conditioning state).

- Finally, reactant $B$ should be supplied into the reactor to start production.

In this study the third step is used as the state of the reactor at time $\sigma=0$, because the details of achieving this operating state are process dependent. Verwijs et al. (1992) demonstrated qualitatively that for an adiabatic tubular reactor a much higher initial reactor temperature is required during startup, compared to the reactor inlet temperature at normal steady-state conditions, to ensure that no excessive amounts of reactant $B$ appear in the reactor effluent during the startup. Therefore, questions to be addressed are: 


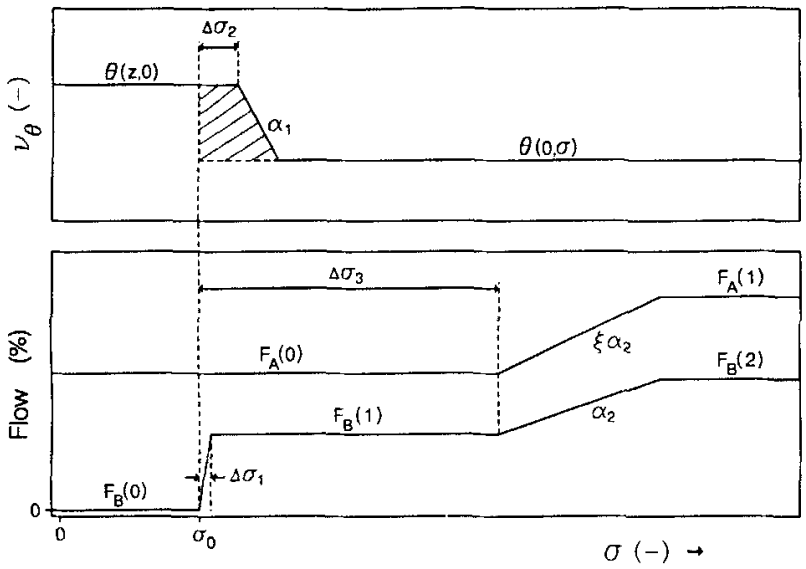

Figure 2. Manipulated variable trajectories during the reactor startup $\left(\sigma_{0}=0.1\right)$.

- What is the appropriate initial temperature $\theta(z, 0)$ at which reactant $B$ can be introduced into the reactor?

- At which total flow rate $\phi_{\nu}$ can the reactor be started up safely?

- How fast can reactant $B$ be introduced into the reactor?

- According to which trajectory should the reactor inlet temperature $\nu_{\theta}$ be decreased from the overheated initial temperature $\theta(z, 0)$ to the normal reactor inlet temperature $\theta(0, \sigma)$ ?

The approach shown in Figure 2 is chosen to study the reactor startup. In the process conditioning state, reactant $A$ is fed at a flow rate $F_{A}(0)$ into the reactor. The reactant $A$ flow is kept at this rate during the startup of the reactant $B$ feed pump. The reactant $B$ feed pump is initially not in service, so the reactant $B$ flow rate $F_{B}(0)=0$. The entire reactor is assumed to be at the required initial temperature level $\theta(z, 0)$. the temperature profile $\theta(z, 0)$ is assumed to be uniform over the entire reactor length $z$. At time $\sigma=\sigma_{0}$ the reactant $B$ feed pump is put into service and the reactant $B$ flow is increased from zero to $F_{B}(1)$ at a constant rate in a period $\Delta \sigma_{1}$ (Figure 2). The flow rate $F_{B}(1)$, or the $F_{A} / F_{B}$ flow ratio, is chosen to conform to the required product mix specification of the process. The reactant $A$ and $B$ flow ratio $\xi$ is kept constant in this study:

$$
\xi=\frac{F_{A}}{F_{B}}=1.15
$$

The reactor inlet temperature $\nu_{\theta}$ has to be decreased from the initial value $\theta(z, 0)$ to the normal reactor inlet temperature $\theta(0, \sigma)$, starting at time $\sigma_{0}+\Delta \sigma_{2}$ at a rate $\alpha_{1}$. The entire process section is driven to the required production capacity target at time $\sigma_{0}+\Delta \sigma_{3}$ by increasing the reactant $B$ flow rate from $F_{B}(1)$ to $F_{B}(2)$ at a rate $\alpha_{2}$. The flow ratio $\xi$ will also be kept constant during this capacity rate increase.

Process dynamics are often studied by controlling the manipulated variables according a step function approach. Such an approach is not used in this study because (major) step changes of manipulated variables cannot be realized in industrial practice. Second, Verwijs et al. (1992) demonstrated that a complete conversion of reactant $B$ during the startup relies on a sufficiently high average reaction rate. The reaction rate, just after the reactant $B$ feed pump startup, is determined by the initial reactor temperature and the concentration of reactant $B$ at the reactor inlet. So, a low reactant $B$ inlet concentration due to an insufficiently fast reactant $B$ flow rate increase may result in an excessive amount of reactant $B$ in the reactor effluent!

The trajectories of the reactant $A$ and $B$ flow rate and the reactor inlet temperature are parameterized by using simple ramp function approximations. This ramp function approach is chosen, because:

- Ramp functions can be implemented relatively easily in modern process control systems

- Transient operations of manipulated variables using a ramp function approach are easy to monitor for the process of safeguarding purposes

- The resulting optimization problem is less time-consuming and much easier to solve than optimization problems involving calculations of multiple parameters trajectories (McAuley and MacGregor, 1992).

The reactor inlet temperature trajectory $v_{\theta}$ is described by

$$
\begin{gathered}
0 \leq \sigma<\left(\sigma_{0}+\Delta \sigma_{2}\right): \\
v_{\theta}=\theta(z, 0) \sigma \geq\left(\sigma_{0}+\Delta \sigma_{2}\right) \wedge \nu_{\theta}>\theta(0, \sigma): \\
\nu_{\theta}=\theta(z, 0)+\alpha_{1}\left[\sigma-\left(\sigma_{0}+\Delta \sigma_{2}\right)\right] \\
\sigma \geq\left(\sigma_{0}+\Delta \sigma_{2}\right) \wedge \nu_{\theta} \leq \theta(0, \sigma): \\
\nu_{\theta}=\theta(0, \sigma) .
\end{gathered}
$$
by

The trajectory of the reactant $A$ flow rate $F_{A}$ is described

$$
\begin{aligned}
& 0 \leq \sigma<\left(\sigma_{0}+\Delta \sigma_{3}\right): \\
& F_{A}=F_{A}(0) \sigma \geq\left(\sigma_{0}+\Delta \sigma_{3}\right) \wedge F_{A}<F_{A}(1): \\
& F_{A}=F_{A}(0)+\alpha_{2} \xi\left[\sigma-\left(\sigma_{0}+\Delta \sigma_{3}\right)\right] \\
& \sigma \geq\left(\sigma_{0}+\Delta \sigma_{3}\right) \wedge F_{A} \geq F_{A}(1): \\
& \quad F_{A}=F_{A}(1) .
\end{aligned}
$$
by

The trajectory of the reactant $B$ flow rate $F_{B}$ is described

$$
\begin{gathered}
0 \leq \sigma \leq \sigma_{0}: \\
F_{B}=F_{B}(0)=0 \\
\sigma_{0}<\sigma<\left(\sigma_{0}+\Delta \sigma_{1}\right) \wedge F_{B}<F_{B}(1): \\
F_{B}=F_{B}(1) \frac{\left(\sigma-\sigma_{0}\right)}{\Delta \sigma_{1}}\left(\sigma+\Delta \sigma_{1}\right) \leq \sigma<\left(\sigma_{0}+\Delta \sigma_{3}\right): \\
F_{B}=F_{B}(1) \sigma \geq\left(\sigma_{0}+\Delta \sigma_{3}\right) \wedge F_{B}<F_{B}(2): \\
F_{B}=F_{B}(1)+\alpha_{2}\left[\sigma-\left(\sigma_{0}+\Delta \sigma_{3}\right)\right] \\
\sigma \geq\left(\sigma_{0}+\Delta \sigma_{3}\right) \wedge F_{B} \geq F_{B}(2): \\
\quad F_{B}=F_{B}(2) .
\end{gathered}
$$




\section{Operating targets and constraints}

Operational problems in the recovery section may easily arise due to an excessive amount of reactant $B$ in the reactor effluent. In this article the term breakthrough is used to characterize this excessive amount of reactant $B$ in the reactor effluent. The operational objective is to minimize the total amount of reactant $B$ fed to the recovery section during the reactor startup. The total amount of reactant $B$ in the reactor effluent is far below the maximum constraint value at normal operating conditions. Therefore, the calculation of the total reactant $B$ breakthrough figure $\Omega_{B}$ can be performed over a fixed time interval $\sigma_{\Delta}$. This time interval is chosen in such a manner that within the possible range of operating conditions the reactant $B$ concentration $\Gamma_{B}(1, \sigma)$ always approaches the normal reactor effluent conditions at time $\sigma_{\Delta}$. The trajectories of the manipulated variables $\nu_{\theta}, \psi_{B}$, and $\phi_{v}$ and the initial reactor temperature $\theta(z, 0)$ will be evaluated against a maximum allowable reactant $B$ breakthrough value $\Omega_{B} \leq 0.45$. This maximum allowable value of $\Omega_{B}$ is based on a safety analysis of the reactant $A$ recovery section, of which the details are beyond the scope of this study.

The reactant $B$ breakthrough value $\Omega_{B}$ is calculated according to:

$$
\Omega_{B}=\chi \int_{0}^{\sigma_{\Delta}} \phi_{\nu} \Gamma_{B}(1, \sigma) d \sigma \leq 0.45
$$

As mentioned before, the initial reactor temperature profile $\theta(z, 0)$ should be overheated compared to the reactor inlet temperature at normal operating conditions. However, the reactor temperature cannot be maintained at the $\theta(z, 0)$ value during the entire startup period, because otherwise the maximum allowable operation temperature of the reactor, $\theta_{\max }=$ 1.36 , will be exceeded. A target value $\theta_{\max }=1.35$ will be used in the design of the reactor startup procedure to incorporate a safety margin vs. this temperature constraint.

The capacity of the reactor feed preheater is also limited. The total flow rate $\phi_{v}$ is constrained by the range of the flow control valve in the reactor effluent line. To simplify the modeling of the startup of the reactant $B$ feed pump, the assumption is made that the reactant $B$ flow rate $F_{B}(1)$ can be established within a time period that equals $\Delta \sigma_{1}$ regardless the value of $F_{B}(1)$; see Figure 2 . The reactant flow increase rate parameters $\Delta \sigma_{1}$ and $\alpha_{2}$, the initial reactor temperature $\theta(z, 0)$, and the reactor inlet temperature decrease rate parameters $\Delta \sigma_{2}$ and $\alpha_{1}$ are bounded for this particular plant as well.

The maximum and minimum constraints of all parameters that can be adjusted to improve the reactor startup operations are listed in Table 2. Note that no constrained values are given for $\alpha_{2}$ and $\Delta \sigma_{3}$ in Table 2 because they are determined by the process dynamics of the entire plant section; these questions are beyond the scope of this study. Nevertheless, the question of when to start increasing the reactant $B$ flow at a rate $\alpha_{2}$ can be answered in view of the previously defined objective of minimizing $\Omega_{B}$.

\section{Simulation and optimization}

The numerical method of lines (Schiesser, 1991) is applied to transform the partial differential equations (Eqs. 1, 2, and
3) into a set of ordinary differential equations; see Verwijs et al. (1992) for details. The resulting set of ordinary differential equations is solved by using a fifth-order, variable step-size, Runge-Kutta-Fehlberg integration algorithm (Steiner et al., 1990b). A generalized-reduced-gradient (GRG) optimization algorithm is used to minimize the objective function $\Omega_{B}$ by varying the initial reactor temperature $\theta(z, 0)$, the time intervals $\Delta \sigma_{1}, \Delta \sigma_{2}$, and the reactor inlet temperature decrease rate $\alpha_{1}$. The total flow rate $\phi_{v}$ is kept constant during a particular optimization run. The maximum temperature constraint $\theta_{\max }$ is taken into account as a global model constraint. All calculations are performed by using the SimuSolv (1993) program running on a DEC 3000 model 500 AXP computer; see Steiner et al., (1990a,b) for details about SimuSolv (1993), and Verwijs et al. (1992) for details about simulation and parameter estimation of this particular plant reactor model.

\section{Results}

The objective function $\Omega_{B}$ is minimized by manipulating the trajectories of $\nu_{\theta}, \psi_{B}$, and $\phi_{\nu}$. The global constraint $\theta_{\max }$ $\leq 1.35$ is taken into account simultaneously. Two key items will determine if the objective $\Omega_{B}(\max ) \leq 0.45$ can be achieved:

- The reaction rate over the entire reactor length $z$, which is a function of the reactant $B$ concentration $\Gamma_{B}$ and the reactor temperature $\theta$.

- The average residence time and residence time distribution of the fluid, which are determined by the superficial fluid velocity $\phi_{v}$ and the Péclet numbers $P e_{m r}$ and $P e_{h r}$ for mass and heat dispersion, respectively. In this article, $P e_{m r}$ and $P e_{h r}$ have the same value for the sake of simplicity.

The Péclet number Pe can be calculated from the model parameters at reference conditions according to:

$$
\begin{aligned}
P e & =\frac{v_{t} L}{D_{a x}}=\left(\frac{v_{t}}{v_{r}}\right)\left(\frac{v_{r} L}{D_{a x}}\right)=\phi_{\nu} P e_{r} \\
P e_{r} & =P e_{m r}=P e_{h r} .
\end{aligned}
$$

A remark should be made about the use of the mass and heat dispersion numbers $P e_{m r}$ and $P e_{h r}$ in Eqs. 1 and 2. In practice, the Péclet number $\mathrm{Pe}$ is almost constant for a specific reactor system because the axial mass and heat dispersion coefficients are usually proportional to the fluid velocity $\phi_{v}$. Nevertheless, the assumption has been made that the dispersion coefficients are constant. This is done to simplify the model transformation from a nondimensionless into a dimen-

Table 2. Minimum and Maximum Values of the Constrained Parameters

\begin{tabular}{ccc}
\hline Parameter & Minimum & Maximum \\
\hline$\theta(z, \sigma)$ & 0.97 & 1.35 \\
$\phi_{v}$ & 0.50 & 1.10 \\
$\theta(z, 0)$ & 0.97 & 1.10 \\
$\Delta \sigma_{1}$ & 0.03 & 0.20 \\
$\Delta \sigma_{2}$ & 0.00 & 0.40 \\
$\alpha_{1}$ & -0.43 & -0.13 \\
\hline
\end{tabular}




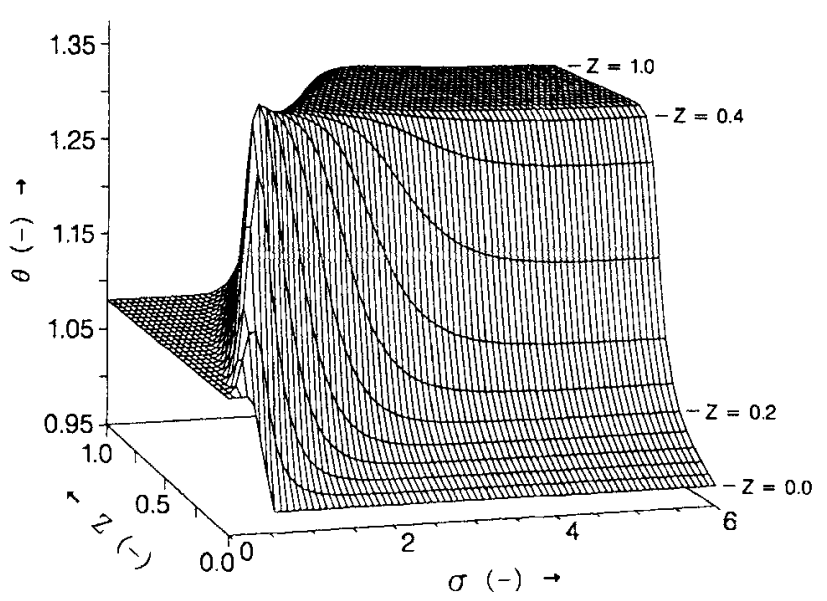

Figure 3. Calculated reactor temperature vs. reactor location and time.

$P e=100, \phi_{u}=0.5, \Delta \sigma_{1}=0.03, \Delta \sigma_{2}=0.201, \alpha_{1}=-0.43$, and $\theta(z, 0)=1$.10. The breakthrough number $\Omega_{B}=0.13$.

sionless form as described in detail by Verwijs et al. (1992). As a result, the Péclet number value $P e$ in the model changes when the fluid velocity is decreased or increased. The impact of the absolute value of the Péclet number Pe on the calculated results will be negligible in view of uncertainties in the process data that are used to control the actual plant start-up operations because the reactor system operates at relatively high Péclet number values of above 30 .

Some modeling results at a superficial fluid velocity $\phi_{\nu}=0.5$ are shown in the Figures 3 and 4. The temperature profile $\theta$ as a function of time $\sigma$ over the entire reactor is shown in Figure 3. In this figure the lines parallel to the $\sigma$-axis represent the temperature $\theta$ at location $z$, and the lines parallel to the $z$-axis connect the data at the same moment. The reactant $B$ concentration $\Gamma_{B}$ is shown in Figure 4 . In this figure the $\sigma$-axis is drawn from the right side to the left, and the $z$-axis is drawn in the opposite direction, which contradicts with the temperature profile in Figure 3. Note that at time

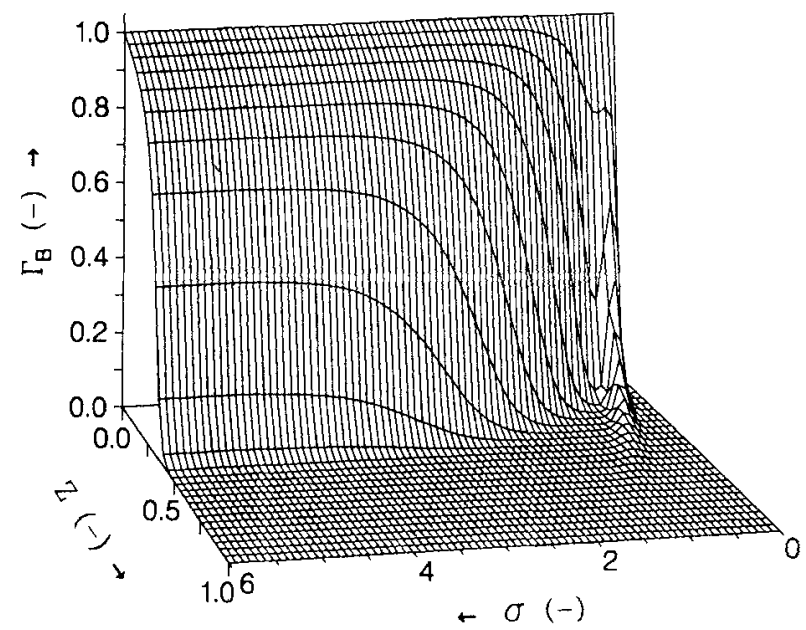

Figure 4. Calculated reactant $B$ concentration vs. reactor location and time.

$P e=100, \phi_{0}=0.5, \Delta \sigma_{1}=0.03, \Delta \sigma_{2}=0.201, \alpha_{1}=-0.43$, and $\theta(z, 0)=1.10$. The breakthrough number $\Omega_{B}=0.13$. $\sigma=0.56$ and location $z=0.12$ the reactor temperature $\theta$ hits the constraint $\theta_{\max }=1.35$ (Figure 3 ). The maximum value of the reactant $B$ concentration in the reactor effluent is approached at time $\sigma=1.5$. The reactant $B$ breakthrough number $\Omega_{B}=0.13$, which is well below the constraint $\Omega_{B} \leq$ 0.45 .

Some results at a superficial fluid velocity $\phi_{\nu}=0.9$ are shown in Figure 5 and 6 . In this case the maximum reactor temperature again hits the constraint $\theta_{\max }=1.35$, but now the breakthrough number is $\Omega_{B}=0.90$, which is unacceptable with respect to the constraint $\Omega_{B} \leq 0.45$. Note the reactant $B$ breakthrough in the reactor effluent at time $\sigma \approx 1$ in Figure 6.

\section{Impact of $\phi_{v}$ and $P e_{r}$}

The objective function $\Omega_{B}$ is calculated at various $\phi_{v}$ and $\mathrm{Pe}_{r}$ numbers to determine the impact of the average residence time and residence time distribution of the fluid. The results are presented in Figure 7. The dashed lines characterize $\Omega_{B}$ as a function of the superficial fluid velocity $\phi_{v}$ and the Péclet number $\mathrm{Pe}$ for a fixed $\mathrm{Pe}_{r}$ value, while the solid lines describe $\Omega_{B}$ as a function of the Péclet number Pe for a fixed $\phi_{v}$ value. The values of the parameters used to diminish the objective function $\Omega_{B}$ followed a specific pattern for the operating conditions considered:

- The initial reactor temperature is always driven to the extreme $\theta(z, 0)=1.10$

- The reactor inlet temperature decrease rate parameter $\alpha_{1}$ is always forced to the constraint $\alpha_{1}=-0.43$, which means that the reactor inlet temperature is driven as fast as possible from the overheated initial reactor temperature $\theta(z, 0)$ to the normal inlet temperature $\theta(0, \sigma)$

- The reactant $B$ flow rate parameter $\Delta \sigma_{1}$ is driven to its minimum value $\Delta \sigma_{1}=0.03$, which means that reactant $B$ is introduced into the reactor as fast as possible

- The time interval $\Delta \sigma_{2}$ is maximized with respect to the global model constraint $\theta_{\max } \leq 1.35$

There are some exceptions to these parameter value trends. At high capacity rates and at high $P e_{r}$ numbers $\left(\phi_{\nu}=0.9\right.$ and

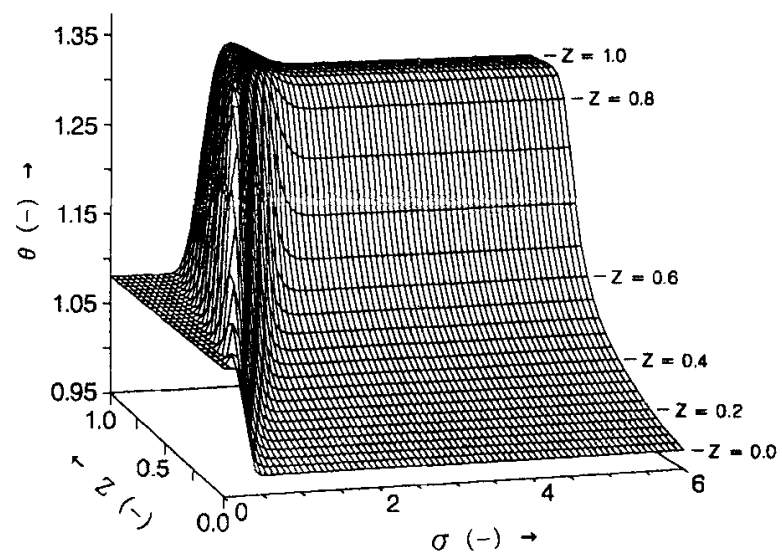

Figure 5. Calculated reactor temperature vs. reactor location and time.

$P e=100, \phi_{u}=0.9, \Delta \sigma_{1}=0.03, \Delta \sigma_{2}=0.057, \alpha_{1}=-0.43$, and $\theta(z, 0)=1.10$. The breakthrough number $\Omega_{B}=0.90$. 


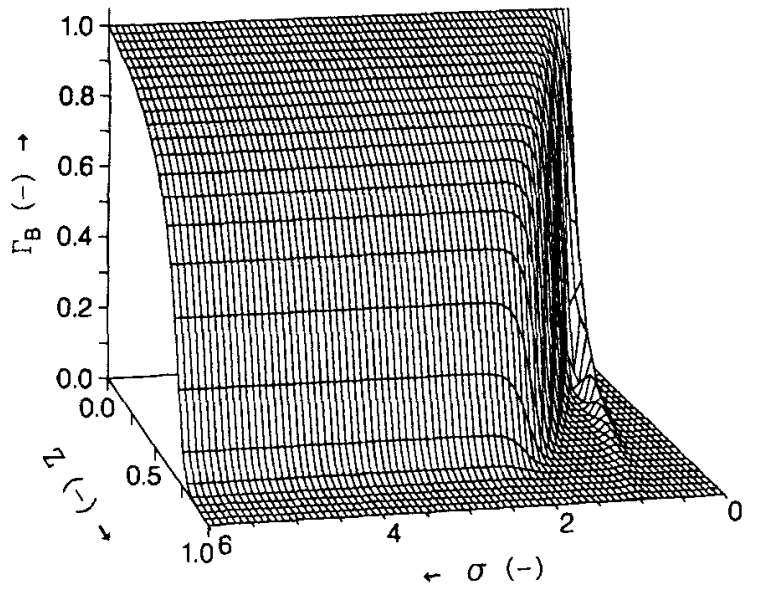

Figure 6. Calculated reactant $B$ concentration vs. reactor location and time.

$P e=100, \phi_{u}=0.9, \Delta \sigma_{1}=0.03, \Delta \sigma_{2}=0.201, \alpha_{1}=-0.43$, and $\theta(z, 0)=1.10$. The breakthrough number $\Omega_{B}=0.90$.

$P e_{r}=125$ or 150 ; and $\phi_{v}=0.8$ and $\left.P e_{r}=150\right)$ the global constraint $\theta_{\max } \leq 1.35$ could not be satisfied by manipulating the time interval $\Delta \sigma_{2}$ only, when the other parameters were set at the previously mentioned constraint values. Therefore, the time interval $\Delta \sigma_{1}$ was manipulated as well in these particular cases. As a result, the minimum achievable value of $\Omega_{B}$ increased slightly in comparison with the figures achieved when the $\Delta \sigma_{2}$ constraint was not a limiting factor (Figure 7).

During startup the frontiers of the reactant $B$ concentration profile and the temperature profile are traveling through the reactor. These frontiers will be elongated by mass and heat dispersion effects. As a result, the temperature rise rate during startup operations will be much slower in a reactor with a relatively low Péclet number in comparison with a reactor system with a relatively high Péclet number. Consequently, the time interval $\Delta \sigma_{2}$ can be increased by decreasing Péclet numbers. On the other hand, mass dispersion effects will promote the transport of reactant $B$ from the reactor inlet to the outlet. The overall result is that for $P e_{r}>75$

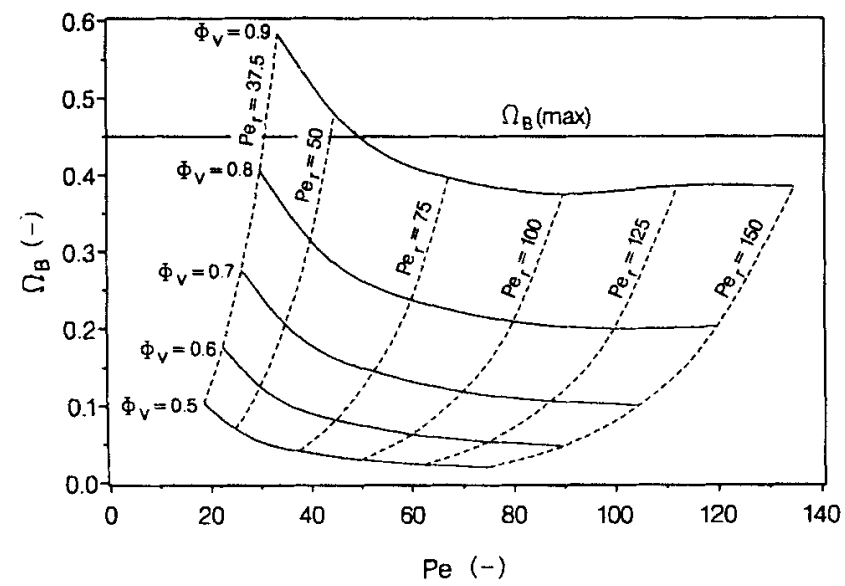

Figure 7. Calculated minimum reactant $B$ breakthrough $\Omega_{B}$ as a function of the Peclet number $P e$, at various superficial fluid velocity $\phi_{v}$. the impact of $P e_{r}$ on $\Omega_{B}$ is negligible compared to the impact of the superficial fluid velocity $\phi_{\nu}$, while below this value the effect of $P e_{r}$ becomes significant, too; see Figure 7.

Two conclusions can be drawn from the results just described:

- To maximize the reaction rate at initial startup conditions reactant $B$ is introduced into the reactor as fast as possible and the initial reactor temperature $\theta(z, 0)$ is driven to its upper extreme simultaneously.

- To compensate for the reaction rate decline by (a) the energy take-up in the reactor vessel, and (b) the elongation of the reactant $B$ concentration profile and the reactor temperature profile due to mass and heat dispersion effects, the reactor should be overheated at initial startup conditions. This additional heat input is determined by the parameters $\theta(z, 0)$, $\Delta \sigma_{2}$, and $\alpha_{1}$, and is indicated by the shaded area in Figure 2 . This excess heat input into the reactor is maximized by taking into account the global model constraint $\theta_{\max } \leq 1.35$.

\section{Impact of $\theta(z, 0)$}

The parameters $\Delta \sigma_{1}, \Delta \sigma_{2}$, and $\alpha_{1}$ are optimized for different values of the initial reactor temperature $\theta(z, 0)$ to quantify the impact of $\theta(z, 0)$ on the minimum value of the objective function $\Omega_{B}$. The calculations are done for a Péclet number $P e_{r}=100$ at several superficial flow rate values $\phi_{v}$. The results are presented in Figure 8.

The initial reactor temperature $\theta(z, 0)$ has a significant impact on the breakthrough number $\Omega_{B}$. The constraint $\Omega_{B}(\max ) \leq 0.45$ may be exceeded very easily due to flow and/or temperature control disturbances when a reactor is started up at high flow rates. Flow and temperature measurement inaccuracies/uncertainties have to be taken into account as well. Verwijs et al. (1992) mentioned that for the plant reactor studied the temperature is measured with an accuracy of $\leq 0.5 \%$. This aspect already makes it not feasible to start up this reactor at a flow rate $\phi_{v}=0.9$. Consequently, it is preferable to start up the reactor at low flow rates in order to stay well below the $\Omega_{B}(\max )$ constraint, since the tolerances for the initial reactor temperature setpoint and the superficial flow target are less confined at low capacity rates.

\section{Impact of $\Delta \sigma_{1}, \Delta \sigma_{2}$, and $\alpha_{1}$}

In the process conditioning step the reactor is operated at a flow rate $F_{A}(0)$, while the reactor temperature is driven to the target $\theta(z, 0)$. This operation as such is independent of the introduction of reactant $B$ into the reactor. This implies that during startup the breakthrough number $\Omega_{B}$ and the maximum reactor temperature $\theta_{\max }$ can be controlled only by the parameter values $\Delta \sigma_{1}, \Delta \sigma_{2}$, and $\alpha_{1}$ ! Consequently, it is very important to operate the reactor properly at the flow and temperature targets at time $\sigma_{0}$, since the initial reactor temperature $\theta(z, 0)$ has a high impact on the breakthrough number $\Omega_{B}$.

In the previous section it is shown that the reactor should be started up at low flow rates in order to stay below the maximum allowable value of $\Omega_{B}$. Therefore, the flow rate is set to $\phi_{v}=0.5$, which implies that according to Eqs. 8 and 10 the reactant $A$ and $B$ flows are controlled at a rate $F_{A}=$ $33.5 \%$ and $F_{B}=29.2 \%$, respectively. These flow rate targets 


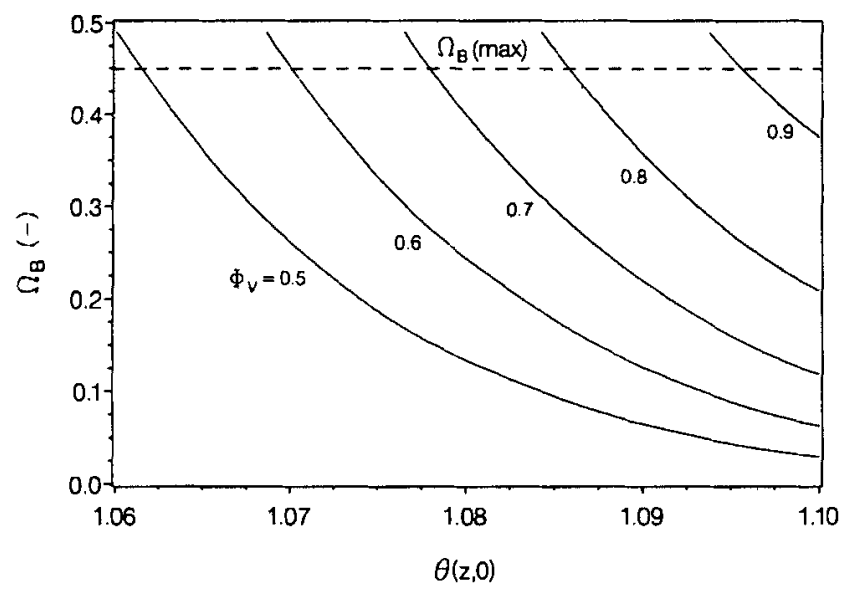

Figure 8. Calculated minimum reactant $B$ breakthrough $\Omega_{B}$ as a function of the initial reactor inlet temperature $\theta(z, 0)$, at various superficial fluid velocity $\phi_{v}\left(P e_{r}=100\right)$.

are well within the controllability limits of the flow control valves in the reactor system.

The parameter values of $\Delta \sigma_{2}$ and $\alpha_{1}$ are varied around the calculated optimum to study their impact on the breakthrough number $\Omega_{B}$ and the maximum reactor temperature $\theta_{\max }$. The parameter $\Delta \sigma_{1}$ is kept at its optimum value $\Delta \sigma_{1}=$ 0.03 and the initial reactor temperature is set to $\theta(z, 0)=1.08$. The results are shown in Figures 9 and 10. It can be concluded from the data presented in Figure 9 that a $10 \%$ variation of the parameters $\Delta \sigma_{2}$ and $\alpha_{1}$ has no significant impact on the breakthrough number $\Omega_{B}$, while Figure 10 shows that such parameter value changes have a relatively small effect on the maximum reactor temperature $\theta_{\text {max }}$.

The impact of variations in the $\Delta \sigma_{1}$ parameter value is studied in a similar way. The parameters $\Delta \sigma_{2}$ and $\alpha_{1}$ are equal to their optimum values, while $P e_{r}=100$ and the initial reactor temperature $\theta(z, 0)=1.08$. The impact of $\Delta \sigma_{1}$ is very limited too. A variance of $30 \%$ to the optimum $\Delta \sigma_{1}$ value

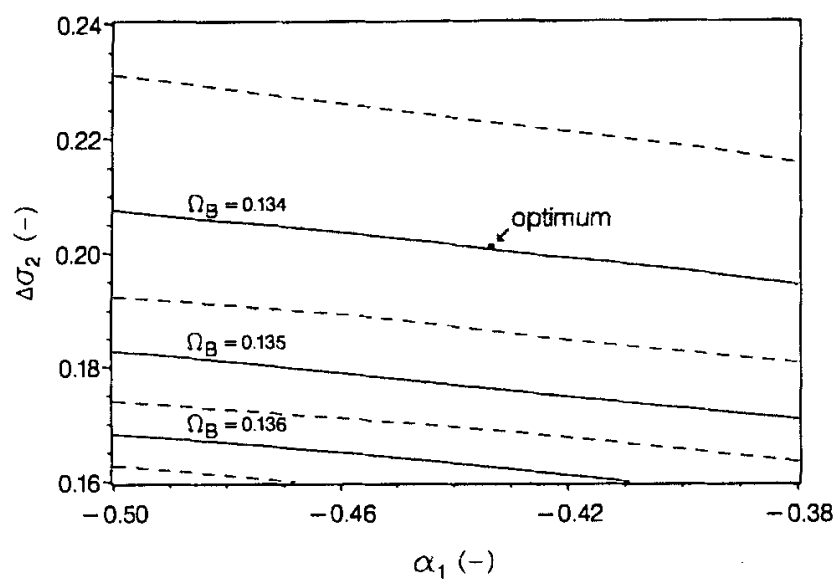

Figure 9. Impact of the parameters $\Delta \sigma_{2}$ and $\alpha_{1}$ on the minimum reactant $B$ breakthrough $\Omega_{B}$.

The calculations are performed for the parameter values $P e_{r}=100, \Delta \sigma_{1}=0.03, \theta(z, 0)=1.08$, and $\phi_{u}=0.5$.

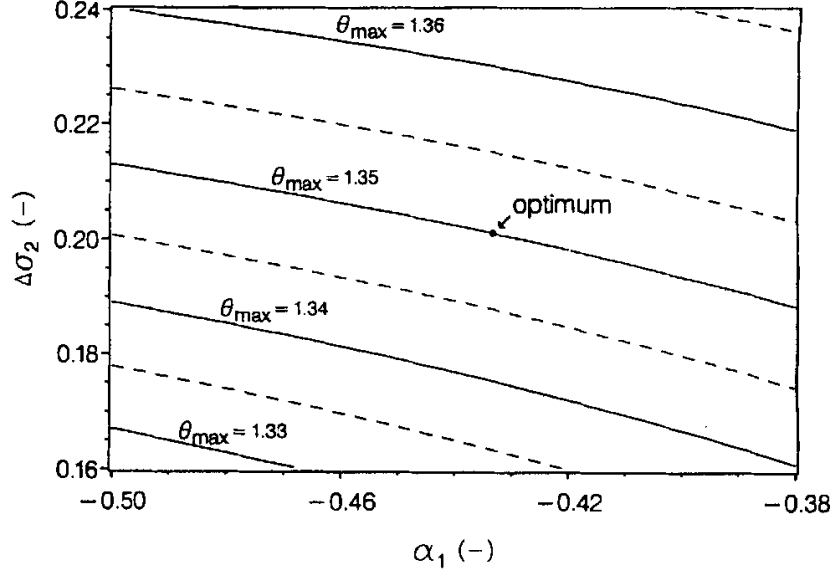

Figure 10. Impact of the parameters $\Delta \sigma_{2}$ and $\alpha_{1}$ on the maximum temperature $\theta_{\max }$ along the reactor length $z$.

The calculations are performed for the parameter values $P e_{r}=100, \Delta \sigma_{1}=0.03, \theta(z, 0)=1.08$, and $\phi_{u}=0.5$.

resulted in a deviation of $0.13 \%$ in the value of $\theta_{\max }$ and $0.4 \%$ in the value of $\Omega_{B}$.

\section{Minimum value of $\Delta \sigma_{3}$}

At time $\sigma_{0}+\Delta \sigma_{3}$ the reactant $B$ flow setpoint will be increased at a rate $\alpha_{2}$ in order to obtain the required production capacity target $F_{B}(2)$ (Figure 2 ). This operation as such should have no impact on the breakthrough number $\Omega_{B}$. In Figure 11, the reactant $B$ concentration $\Gamma_{B}$ in the reactor effluent is shown as a function of time, at different values of the superficial fluid velocity $\phi_{v}$. The breakthrough number $\Omega_{B}$ is indicated as well for the various conditions.

The reactant $B$ concentration in the reactor effluent returns to the normal operating conditions in the time interval $1.3<\sigma<2$, depending on the flow rate $\phi_{\nu}$. The amount of reactant $B$ in the reactor effluent is well below the critical limit at normal operating conditions. So, after a period of time equal to $\sigma=2$, the flow can be increased without affecting the breakthrough number $\Omega_{B}$, regardless of the flow rate value $\phi_{v}$. A time difference of about $\Delta \sigma=0.7$ is normally not significant in view of the entire startup time of a continuously operated chemical plant. Hence, the recommendation for this particular reactor system is to increase the flow rate at time $\sigma=2$ or later in order to exclude all risks for a reactant $B$ breakthrough in the reactor effluent due to a flow rate increase during startup operations.

A remark should be made about the location of the maxima of the $\Gamma_{B}$ functions presented in Figure 11. The maximum of the function $\Gamma_{B}$ is not located at $\sigma=1$ at a flow rate $\phi_{\nu}=1$, due to the choice of the value of the fluid velocity at reference conditions $\left(v_{r}\right)$, which is used to scale the dimensionless flow rate $\phi_{v}$.

\section{Startup strategy design principles}

It can be concluded from the results presented in the Figures $7,8,9$, and 10 that the objective function $\Omega_{B}$ is controlled mainly by the initial reactor temperature $\theta(z, 0)$, while the maxi- 


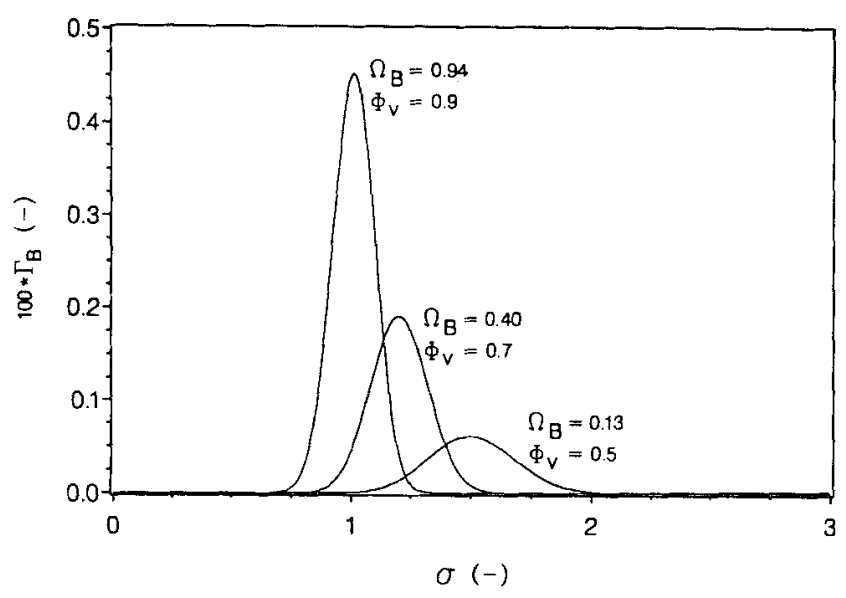

Figure 11. Reactant $B$ concentration in the reactor effluent at various superficial fluid velocities $\phi_{v}$.

The calculations are performed for the parameter values $P e_{r}=100, \Delta \sigma_{1}=0.03, \theta(z, 0)=1.08$

mum allowable reactor temperature $\theta_{\max }$ is controlled primarily by the parameters $\Delta \sigma_{2}$ and $\alpha_{1}$. This is an important result, because now it is possible to design a feasible reactor startup strategy. As said before, in the process conditioning step the reactor is driven to the initial reactor temperature $\theta(z, 0)$. This operation as such is independent of the introduction of reactant $B$ into the reactor, and can be carried out very easily in a properly designed process (Verwijs et al., 1995a). For the reactor system considered, the impact of the parameters $\Delta \sigma_{1}, \Delta \sigma_{2}$, and $\alpha_{1}$ on the breakthrough number $\Omega_{B}$ and the maximum allowable reactor temperature $\theta_{\text {max }}$ is relatively small, when compared to the impact of the initial reactor temperature $\theta(z, 0)$. This implies that deviations from the optimized manipulated variable trajectories, due to process upsets and/or disturbances, can be allowed to a certain extent as long as the reactor inlet temperature condition is satisfied.

Steady-state and dynamic operation optimization of production facilities is one of the major challenges for the chemical industry to strengthen profitability. Steady-state process optimization is reported to contribute roughly $3-5 \%$ to the profit margin of continuously operated chemical plants (Cutler and Perry, 1983; Baily et al., 1993; Krist et al., 1994). Dynamic simulation and optimization of process operations is a fairly new activity, but is at least of the same economic importance to the chemical industry. By applying the presented startup methodology to the specific production facility studied, both the startup time (order of magnitude: days) as well as the amount of off-grade material produced during startup (order of magnitude: 100 metric tonne) could be reduced by about $75 \%$ compared to the figures that resulted from the startup methodology described by Verwijs et al. (1992).

\section{Reactor Safeguarding}

In general, a process is operated and safeguarded by controlling the process inventory, material flows, temperatures, and pressures within certain operating windows (Verwijs et al., 1995a,b). Other safeguarding items like rotating equipment control, valve position control, and on-line process analyzers may be used as well in a chemical plant. When the value of a process state variable exceeds a certain limit, a corrective action is taken by the process control system or by the operating personnel. Usually, such corrective actions are taken automatically at emergency conditions by hard-wired trip or safety control systems, because computerized systems are said to be not sufficiently reliable for use as emergency shutdown systems (Barton and Rogers, 1993).

This safeguarding strategy is normally sufficient for process units in which no (exothermic) chemical reaction is carried out, but it may (easily) fail for chemical reactors. Verwijs et al. (1995b) demonstrated the inadequacy of this safeguarding strategy by using experimental data of runaways that occurred in an industrial adiabatic tubular reactor. The reason for this imperfection is that the mass and heat balances over the reactor are uncoupled by controlling the reactant flows and some reactor temperatures as such within certain operating windows, because the temperature of, for example, a tubular reactor is highly sensitive to the reactant feed ratio's and the reactor inlet's temperature. As a result of using operating windows in the safeguarding system, no adequate on-line information is available about the extent of the reaction taking place. Consequently reactants can accumulate relatively easily in the system, especially at abnormal process conditions.

To avoid an unexpected reactant accumulation in the system the temperature profile along the entire reactor length has to be included in the reactor safeguarding strategy (Verwijs et al., 1995b), despite the fact that the spatially distributed nature of the process conditions over the entire reactor length complicates considerably the implementation of a simple control and safeguarding system. Therefore, a model-based process control and safeguarding system is required to control the reactor mass balance in conjunction with the reactor heat balance. In this way, on-line information can be obtained from the model about the extent of the reaction, even at abnormal process conditions.

\section{Safeguarding during startup operations}

The spatially distributed nature of the process conditions in a tubular reactor makes the development of an efficient safeguarding system at steady-state conditions complex, but the situation is even worse for the dynamic case. This can be explained by focusing on the steady-state reactor temperature profile shown in Figure 12. At steady-state conditions the reactor is safeguarded by monitoring the reactor inlet temperature $\left(\Delta \theta_{\text {in }}\right)$, the isothermal operation at the tail end of the reactor $\left(\Delta \theta_{\text {iso }}\right)$, and the adiabatic temperature rise $\left(\Delta \theta_{\mathrm{adr}}\right)$. Additionally, the reactor temperature is observed vs. a maximum and minimum temperature constraint $\left(\theta_{\max }\right.$ and $\left.\theta_{\min }\right)$. The safeguarding elements $\Delta \theta_{\text {iso }}$ and $\Delta \theta_{\text {adr }}$ are initially of no use when reactant $B$ is introduced into the system to start up the reactor, because the normal temperature profile does not exist. Therefore in industrial practice such safeguarding elements are not taken care of during the start-up because they may generate unreasonable reactor shutdowns.

Hence, during startup the plant reactor is safeguarded with respect to mass flow by monitoring:

- The mass balance over the entire reactor by comparing the mass flow of the reactant feed streams with the reactor 


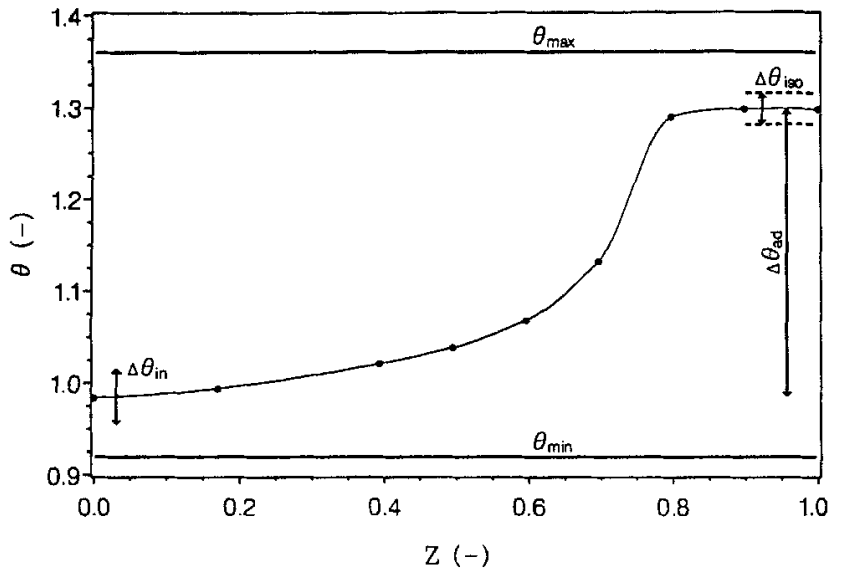

Figure 12. Observed steady-state temperature profile along the reactor length $z$.

Indicated as well are the reactor temperature safeguarding items used at steady-state conditions.

effluent stream, and dependent on the operating mode by monitoring:

- The actual reactant $A$ flow rate into the reactor vs. a flow setpoint, as long as no reactant $B$ is fed into the reactor, or

- The actual reactant $B$ flow rate into the reactor vs. the flow setpoint, and

- The actual flow ratio of reactants $A$ and $B$ into the reactor vs, the flow-ratio setpoint, when the reactant $B$ feed pump is running. Additionally, the reactor is safeguarded with respect to reactor temperature by supervising:

- The reactor inlet temperature vs. the reactor inlet temperature setpoint $\left(\Delta \theta_{\text {in }}\right)$

- The reactor temperature, at all thermoelement locations, vs. a maximum and a minimum allowable reactor temperature constraint ( $\theta_{\max }$ and $\theta_{\min }$ ).

Now, all safeguarding items that may give some indication about the extent of the reaction are missing, and operational problems may easily arise during the startup, as has been demonstrated by Verwijs et al. (1992).

In general, the observation of a few key state variables is often sufficient for experienced operating personnel to control the process status at steady-state conditions (Verwijs et al. 1995a). A tubular reactor is supervised in the same way by the operating personnel. They use the readings of the reactant feed rates together with the values of some thermoelements to control the status of the reactor. The spatially distributed nature and the transient behavior of the temperature readings along the reactor makes it significantly more complicated for plant operating personnel, in comparison with reactor safeguarding at steady-state conditions, (a) to identify important process deviations during dynamic operations, and (b) to recognize when a process is running into hazardous situations. As mentioned in the introduction, the incident frequency of continuously operated chemical plants is, on a time basis, much higher during startup operations than at normal steady-state conditions. It is likely that the imperfection of the currently practiced safeguarding strategy has a significant impact on this high-incident frequency during startup operations. A model-based control system, based on the reactor mass and heat balance, can provide a solution to this problem.

\section{Improved safeguarding strategy}

One method of performing model-based process control and safeguarding is to solve the full nonlinear dynamic model on-line at each control interval by using process measurements to provide information about disturbances and model mismatch. This methodology is often not feasible due to restrictive computing power (McAuley and MacGregor, 1993; Terwiesch and Agarwal, 1994).

Another methodology is to use off-line optimized trajectories for the manipulated variables, given the dynamic model and the initial reactor conditions. During the startup, on-line information about deviations from the computed optimal trajectories becomes available, and normally the actual input profiles must be adapted to preserve optimality. Terwiesch and Agarwal (1994) developed a neighboring extremal technique for on-line adaptation of the preoptimized input profiles of semibatch reactors that requires little computing power. However, the on-line correction of the input profiles is disabled at the beginning of the batch, because insufficient information is available about deviations from the nominal path at that time to perform such corrections. It is shown in the previous sections that for the case studied the initial period of the start-up operations is critical to satisfy the objectives $\Omega_{B}(\max ) \leq 0.45$ and $\theta_{\max } \leq 1.35$, while later on the impact of the manipulated variables on these objectives is relatively small. Hence, the neighboring extremal technique is not applicable to the case studied.

Conventionally, the reactor is safeguarded by supervising the instantaneous values of the process state variables within a certain operating window. This methodology is not sufficient for chemical reactor systems, because all items that may give some information about the extent of the reaction are missing during transient operations. Another shortcoming is that this methodology is not suitable for identification of upcoming hazardous process conditions during process transients. Therefore, an improved safeguarding methodology should provide a procedure (a) to control the manipulated variables, and (b) to supervise the resulting dynamic reactor behavior.

This functionality can be introduced by controlling the actual manipulated variable trajectories vs. the optimal trajectories calculated by the model. Subsequently, maximum allowable deviations from the optimal trajectories have to be determined to compensate for process disturbances and model mismatch. In an analgous way, the actual responses of the thermoelements located along the reactor can be monitored vs. the optimal trajectories calculated by the reactor model, and constraints can also be defined to compensate for process disturbances and model mismatch. Now the operating personnel have a method to supervise the dynamic reactor behavior vs. a target response. Consequently, the reactor should be brought to shutdown when these constraints are exceeded to prevent the process from running into a hazardous situation.

The calculated temperature trajectory constraints are represented by the dashed lines in Figure 13 at several positions $z$ along the reactor. These results are obtained by varying the initial reactor temperature $\theta(z, 0)$, starting from the optimal conditions at $\theta(z, 0)=1.08$. All parameter values used are shown in Table 3 . The upper constraints are determined by the maximum allowable reactor temperature $\theta_{\max } \leq 1.36$. Note 

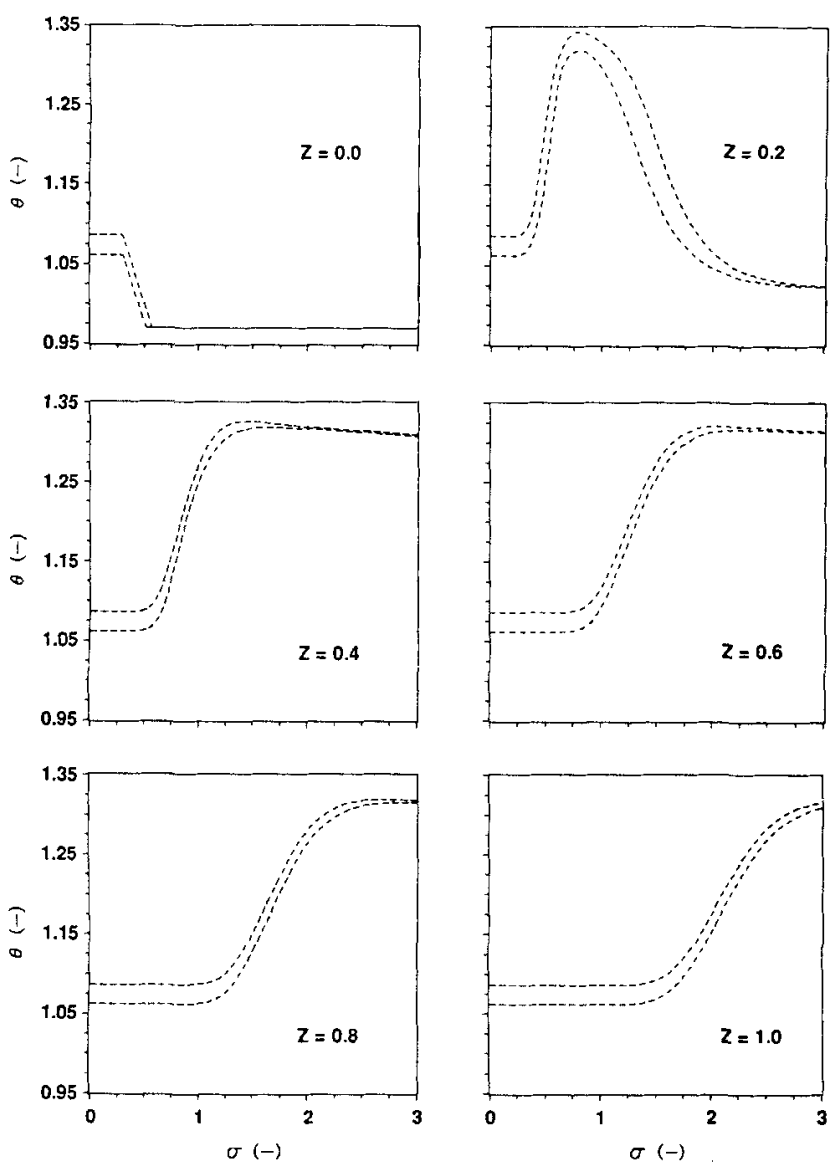

Figure 13. Reactor temperature trajectory constraints for thermoelements located at several positions along the reactor length $z$.

that in this case the maximum allowable reactor temperature constraint is used, and not the temperature constraint $\theta_{\max } \leq$ 1.35, which is used for the optimization calculations. The lower temperature constraint is determined by the maximum allowable breakthrough number $\Omega_{B}(\max ) \leq 0.45$. So, the temperature range available between these constraints is available for a safe reactor startup.

The methodology just described is also applicable to the design of a reactor system. Now, the maximum allowable reactor temperature $\theta_{\max }$ and the maximum initial reactor temperature $\theta(z, 0)$ can be used as design parameters to enlarge the range between the upper and lower temperature constraints. Enlarging this temperature range will decrease the impact of process disturbances and model mismatch on process safety during the reactor startup.

Table 3. Parameter Values for Manipulated Variable Trajectories and Constraints

\begin{tabular}{cccc}
\hline Parameter & $\begin{array}{c}\Omega_{B}(\max ) \\
\text { Constraint }\end{array}$ & $\begin{array}{c}\text { Optimal Startup } \\
\text { Trajectory }\end{array}$ & $\theta_{\max }$ Constraint \\
\hline$\Delta \sigma_{1}$ & 0.030 & 0.030 & 0.030 \\
$\Delta \sigma_{2}$ & 0.201 & 0.201 & 0.201 \\
$\alpha_{1}$ & -0.43 & -0.43 & -0.43 \\
$\theta(z, 0)$ & 1.062 & 1.080 & 1.086 \\
$\phi_{v}$ & 0.5 & 0.5 & 0.5 \\
$\theta_{\max }$ & 1.321 & 1.350 & 1.360 \\
$\Omega_{B}$ & 0.450 & 0.134 & 0.085 \\
\hline
\end{tabular}

In practice, the period of time up to the first maximum in the temperature trajectories is of major interest for safeguarding the reactor start-up. After this period, the conventional safeguarding system can take over when no on-line model-based control and safeguarding system is available. For this particular period of time the expected thermoelement responses and their related constraints can be characterized very well by the equations that describe the step response of a second-order system with delay and complex conjugate roots of the characteristic equation (Kuo, 1991). So, for this period of time the reactor temperature $\theta(z, \sigma)$ at a particular location $z$ can be described by

$$
0 \leq \sigma<\sigma_{d}: \quad \theta(z, \sigma)=\theta(z, 0)
$$

and for $\sigma \geq \sigma_{d}$

$$
\begin{gathered}
\theta(z, \sigma)=\theta(z, 0)+K\left\{1-\frac{\exp \left[-\beta \omega_{0}\left(\sigma-\sigma_{d}\right)\right]}{\alpha}\right. \\
\left.\sin \left[\theta \omega_{0}\left(\sigma-\sigma_{d}\right)+\varphi\right]\right\} \\
\alpha=\sqrt{1-\beta^{2}} \\
\varphi=\cos ^{-1} \beta \\
\end{gathered}
$$

The parameters $K, \beta, \omega_{0}$, and $\sigma_{d}$ can be used to fit Eq. 18 to the data calculated by the dynamic reactor model described by Eqs. 1 to 6. For reactor startup safeguarding purposes, Eqs. 17 and 18 can be implemented fairly easily in modern process control systems.

\section{Conclusions}

Controlling chemical reactions and their associated hazards is an important aspect of chemical manufacturing processes, since the consequences of such incidents may be dramatic due to the resulting loss of human life and/or the scale of property damage. Dynamic reactor operations in particular are a major area of concern, as can be learned from accident statistics.

The process safeguarding methodology used in the current industrial practice is based on controlling the instantaneous values of the process state variables within certain operating windows. If the safety constraints are exceeded, the process is brought to shutdown. This safeguarding strategy does not necessarily prevent a reactor from a runaway, because excessive amounts of unreacted chemicals can still accumulate in the reactor system. A model-based control and safeguarding system, which controls the reactor mass balance in conjunction with the reactor heat balance, is required to overcome the limitations of the conventional safeguarding system. In this way, on-line information can be obtained from the model about the extent of the reaction, even at abnormal process conditions.

A dynamic model of an industrial adiabatic tubular reactor is used to develop such an improved safeguarding strategy for startup operations. Two safety constraints are taken into account in this study: (a) one of the main reactants has to be 
totally converted at the reactor exit to avoid hazardous situations in a downstream process section, and (b) the reactor temperature should never exceed a maximum allowable reactor temperature constraint. The trajectories of the manipulated variables are parameterized by using a simple ramp function approach, and are used to minimize the breakthrough of the critical component in the reactor effluent and to keep the reactor temperature below the maximum temperature constraint. It is concluded for the operating conditions studied that a proper control of the initial reactor temperature is critical for a safe reactor startup. The impact of other manipulated variables on the safety targets is relatively small in comparison with the effect of the initial reactor temperature. It was also concluded that the reactor should be started up at low capacity rates.

Dynamic operation optimization of production facilities is one of the major challenges for the chemical industry to strength profitability. By applying the presented startup methodology to the specific production facility studied, both the startup time as well as the amount of off-grade material produced during startup could be reduced by about $75 \%$ compared to the figures that resulted from the startup methodology described by Verwijs et al. (1992).

The optimum initial reactor temperature conditions and manipulated variable trajectories are used to determine a range of operating conditions in which the reactor can be started up safely. It is shown that the temperature trajectories measured by the particular thermoelements located along the reactor can be fairly well described by the response of a second-order system to a step response. These equations can be implemented relatively easily in modern process control systems for process safeguarding purposes. Additionally, operating personnel can use such target trajectories to identify upcoming hazardous situations during startup operations.

\section{Notation}

$C_{i}=$ concentration of species $i, \mathrm{~mol} / \mathrm{m}^{3}$

$C_{i 0}=$ concentration of species $i$ at reactor inlet, $\mathrm{mol} / \mathrm{m}^{3}$

$C_{i r}=$ reference concentration of species $i, \mathrm{~mol} / \mathrm{m}^{3}$

$C_{p}=$ specific heat of reaction mixture, $\left.\mathrm{J} / \mathrm{kg} \cdot \mathrm{K}\right)$

$C_{p w}=$ specific heat of reactor vessel material, $\left.\mathrm{J} / \mathrm{kg} \cdot \mathrm{K}\right)$

$d_{1}=$ inside tube diameter, $\mathrm{m}$

$d_{2}=$ outside tube diameter, $\mathrm{m}$

$D a_{r}=\left\{k_{r} L / v_{r}\right\} ;$ Damköhler number

$D_{a x}=$ mass dispersion coefficient, $\mathrm{m}^{2} / \mathrm{s}$

$E_{a}=$ activation energy, $\mathrm{J} / \mathrm{mol}$

$F_{A}(1)=$ flow rate of reactant $A$ at production capacity target conditions, \%

$\Delta H_{r}=$ reaction enthalpy, $\mathrm{J} / \mathrm{mol}$

$\left.k_{1}=\left\{k_{10} \exp \left[-E_{a} \bigwedge R \cdot T\right)\right]\right\}$; reaction rate constant, $1 / \mathrm{s}$

$k_{10}=$ preexponential factor, $1 / \mathrm{s}$

$k_{r}=$ reference reaction rate constant, $1 / \mathrm{s}$

$L=$ reactor length, m

$R=$ gas constant, $\mathrm{J} /(\mathrm{mol} \cdot \mathrm{K})$

$t=$ time, $\mathrm{s}^{(\mathrm{k})}$

$T=$ fluid temperature, $\mathrm{K}$

$T_{0}=$ fluid temperature at reactor inlet, $\mathrm{K}$

$T_{r}=$ reference temperature, $\mathrm{K}$

$T_{w}^{r}=$ reactor wall temperature, $\mathrm{K}$

$U=$ heat transfer coefficient, $\mathrm{W} /\left(\mathrm{m}^{2} \cdot \mathrm{K}\right)$

$\left.U^{*}=\left\{4 U / k_{r} \rho C_{p} d_{1}\right)\right\}$, dimensionless heat-transfer coefficient

$v_{t}=$ superficial fluid velocity, $\mathrm{m} / \mathrm{s}$

$x=$ length coordinate in direction of flow, $\mathrm{m}$

$\gamma=\left\{E_{a} \wedge\left(R \cdot T_{r}\right)\right\}$; dimensionless activation temperature
$\Gamma_{B 0}=\left\{C_{B 0} / C_{B}\right\} ;$ dimensionless concentration reactant $B$ at the reactor inlet

$\kappa=\left\{k_{1} / k_{r}=\exp [\gamma(1-1 / \theta)]\right\} ;$ dimensionless reaction rate constant

$\lambda_{a x}=$ heat dispersion coefficient, $\mathrm{W} /(\mathrm{m} \cdot \mathrm{K})$

$\rho=$ fluid density, $\mathrm{kg} / \mathrm{m}^{3}$

$\rho_{w}=$ density construction material reactor vessel, $\mathrm{kg} / \mathrm{m}^{3}$

$\sigma_{0}=\left\{\sigma_{0}=0.1\right\}$; point in time at which reactant $B$ is introduced into the reactor

$\Delta \sigma_{3}=$ delay period before the flow rate is increased to the required capacity target

$\chi=\{\chi=1,000\}$; scaling parameter for $\Omega_{B}$

$\omega_{h}=\left\{\rho C_{p} d_{1}^{2} \Lambda \rho_{w} C_{\rho w}\left(d_{2}^{2}-d_{1}^{2}\right)\right\} ;$; dimensionless heat capacity ratio of the reaction mixture to the reactor wall material

\section{Literature Cited}

Bailey, J. K., A. N. Hrymak, S. S. Treiber, and R. B. Hawkins, "Nonlinear Optimization of a Hydrocracker Fractionation Plant," Comput. Chem. Eng., 17, 123 (1993).

Barton, J. A., and P. F. Nolan, "Runaway Reactions in Batch Reactors," Ind. Chem. Eng. Symp. Ser., No. 85, p. 13 (1984).

Barton, J. A., and P. F. Nolan, "Incidents in the Chemical Industry Due to Thermal-Runaway Chemical Reactions," Proc. Conf. and Exhibition on Techniques for Assessment of Chemical Reaction Hazards, London Press Centre, London ECI, IBC Technology Press, London, p. 1 (1989).

Barton, J. A., and R. L. Rogers, eds., Chemical Reaction Hazards-A Guide, Ind. Chem. Eng., Rugby, UK (1993).

Bauman, E., A. Varma, J. Lorusso, M. Dente, and M. Morbidelli, "Parametric Sensitivity in Tubular Reactors with Co-Current External Cooling," Chem. Eng. Sci., 45, 1301 (1990).

Bhola, S. M., "The Role of Inspection and Certification of Chemical Plant Equipment in the Control of Accident Hazards," Symp. Proc. on The Chemical Industry after Bhopal, p. 255 (1985).

Cutler, C. R., and R. T. Perry, "Real Time Optimization with Multivariable Control is Required to Maximize Profits," Comput. Chem. Eng., 7, 663 (1983).

Etchells, J. C., "Prevention and Control of Exothermic Runaway," Proc. Conf., Assessment and Control of Chemical Reaction Hazards, IBC, London (1993).

Garrison, W. G."“Major Fires and Explosions Analyzed for 30-Year Period," Hydrocarbon Processing, 67(9), 115 (1988).

Hasstrup, P., "Design Error in the Chemical Industry," Ind. Chem. Eng. Symp. Ser., Loss Prevention and Safety Promotion, 1(80), J15 (1983).

Hosten, L. H., and G. F. Froment, "Parametric Sensitivity in CoCurrently Cooled Tubular Reactors," Chem. Eng. Sci., 41, 1073 (1986).

Krist, J. H. A., M. R. Lapère, S. Groot Wassink, R. Neyts, and J. L. A. Koolen, "Generic System for On-Line Optimization and the Implementation in a Benzene Plant," Comput. Chem. Eng., Suppl., 18, S517 (1994).

Kuo, B. C., Automatic Control Systems, 6th ed. Prentice-Hall, Englewood Cliffs, NJ (1991).

Marrs, G. P., F. P. Lees, J. Barton, and N. Scilly, "Overpressure Protection of Batch Chemical Reactors," Chem. Eng. Res. Dev., 67, 381 (1989).

McAuley, K. B., and J. F. MacGregor, "Optimal Grade Transitions in a Gas Phase Polyethylene Reactor," AIChE J., 38, 1564 (1992).

McAuley, K. B., and J. F. MacGregor, "Nonlinear Product Property Control in Industrial Gas-Phase Polyethylene Reactors," AIChE J., 39, 855 (1993).

Morbidelli, M., and A. Varma, "A Generalized Criterion for Parametric Sensitivity: Application to a Pseudohomogeneous Tubular Reactor with Consecutive or Parallel Reactions," Chem. Eng. Sci., 44, 1675 (1989).

Nolan, P. F., and J. A. Barton, "Some Lessons from Thermal-Runaway Incidents," J. Hazardous Mater., 14, 233 (1987).

Raghaven, K. V., "Temperature Runaway in Fixed-Bed Reactors: Online and Offline Checks for Intrinsic Safety,"J. Loss Prev. Process Ind., 5, 153 (1992).

Schiesser, W. E., The Numerical Method of Lines. Integration of Partial Differential Equations, Academic Press, San Diego (1991). 
SimuSolv-Modeling and Simulation Software, Version 3.0-124, The Dow Chemical Company, Midland, MI (1993).

Steiner, E. C., T. D. Rey, and P. S. McCroskey, SimuSolv Reference Guide, Vol, 1, The Dow Chemical Company, Midland, MI (1990a). Steiner, E. C., T. D. Rey, and P. S. McCroskey, SimuSolv Reference Guide, Vol. 2, The Dow Chemical Company, Midland, MI (1990b).

Terwiesch, P., and M. Agarwal, "Online Correction of Pre-Optimized Input Profiles for Batch Reactors," Comput. Chem. Eng., 18, S433 (1994).

Verwijs, J. W., H. van den Berg, and K. R. Westerterp, "Start-up of an Industrial Adiabatic Tubular Reactor," AIChE J., 38, 1871 (1992).

Verwijs, J. W., P. H. Kösters, H. van den Berg, and K. R. Westerterp, "Reactor Operating Procedures for Start-up of Continuously-Operated Chemical Plants," AIChE J., 41, 148 (1995a).

Verwijs, J. W., H. van den Berg, and K. R. Westerterp, "Start-up and Safeguarding of an Industrial Adiabatic Tubular Reactor," Chem. Eng. Sci., 49, 5519 (1995b).

Manuscript received Apr. 22, 1994, and revision received Feb. 21, 1995. 